\title{
Saint-Exupéry, à lire et relire
}

\section{Jean Martin}

Dr méd., membre de la rédaction

\author{
On peut toujours bien sûr, d'Antoine de Saint-Exupéry, relire Le Petit Prince - dont \\ on dit qu'il est le deuxième ouvrage le plus traduit au monde après la Bible. Je viens \\ de relire Terre des hommes, publié en 1939 peu avant la guerre qui le fera mourir \\ (disparu en Méditerranée). On sait sa finesse dans la compréhension des rapports \\ humains. Avant lui comme après lui, beaucoup ont aussi cherché à dire ce qui \\ compte et ce qui compte moins. Avons-nous beaucoup appris?
}

Grandeur d'un métier, unir les hommes. «Telle est la morale que Jean Mermoz et d'autres nous ont enseignée. La grandeur d'un métier est d'unir les hommes: il n'est qu'un luxe véritable, et c'est celui des relations humaines. En travaillant pour les seuls biens matériels, nous bâtissons notre prison, solitaires, avec notre monnaie de cendre. Si dans mes souvenirs je fais le bilan des heures qui ont compté, à coup sûr je retrouve celles que nulle fortune ne m'eût procurées.»

L'homme et les techniques. "Si nous croyons que la machine abîme l'homme, c'est que peut-être nous manquons de recul pour juger de transformations aussi rapides que celles que nous avons subies. [...] Chaque progrès nous a chassés hors d'habitudes que nous avions à peine acquises. Nous sommes de jeunes barbares que nos jouets neufs émerveillent encore.» Qu'en dire, à l'heure des interrogations autour d'autres nouvelles machines, de Big Data, de l'intelligence artificielle?

Atteindre la perfection? Intéressant propos sur le progrès techn(olog)ique: "Tout l'effort industriel de l'homme, tous ses calculs, semblent n'aboutir qu'à la seule simplicité, comme s'il fallait l'expérience de plusieurs générations pour dégager peu à peu la courbe d'une colonne ou d'un fuselage, jusqu'à leur rendre la pureté d'un sein ou d'une épaule. [...] La perfection serait atteinte, non quand il n'y a plus rien à ajouter, mais quand il n'y a plus rien à retrancher.» Avec une conclusion qui laisse songeur: «La perfection de l'invention confine ainsi à l'absence d'invention"...

Responsabilité. Il insiste souvent sur la responsabilité de chacun, fort ou faible, quelles que soient ses circonstances. Parlant des équipes de pilotes et d'autres, dans leurs métiers risqués de l'Aéropostale - ou dans un engagement de guerre: "Chacun est seul responsable. Chacun est seul responsable de tous.»

Apporter sa contribution. Importance pour St-Ex de dépasser son intérêt propre et celui du moment pré- sent. «N'espère rien de l'homme s'il travaille pour sa propre vie et non pour son éternité.» "Quiconque lutte dans l'unique espoir de biens matériels ne récolte rien qui vaille de vivre.» «Etre homme, c'est sentir, en posant sa pierre, que l'on contribue à bâtir le monde.» Plus loin: "Quand nous prendrons conscience de notre rôle, même le plus effacé, alors seulement nous pourrons vivre en paix et mourir en paix.» Des idées dont la considération ne nuirait pas aujourd'hui.

Sur la justice sociale: «Il faut exiger de chacun ce que chacun peut donner.»

Aimer. La formule fameuse: «Aimer, ce n'est pas se regarder l'un l'autre, c'est regarder ensemble dans la même direction", appliquée souvent au couple (à qui bien sûr elle peut s'appliquer), évoque une nuit que St-Ex journaliste a passée, pendant la guerre d'Espagne, avec des soldats qui vont lancer une attaque dans laquelle il est probable qu'ils mourront - il le dit aussi d'une cordée d'alpinistes.

Je ne regrette rien. Perdus son mécanicien et lui dans le désert de Libye, après y avoir capoté le 30 décembre 1935, ils marchent plusieurs jours avant de rencontrer un Bédouin. Décrivant ses états d'âme alors qu'il craignait de mourir: "A part votre souffrance [s'adressant à ses proches], je ne regrette rien. J'ai eu la meilleure part. Si je rentrais, je recommencerais. L'avion n'est pas une fin, c'est un moyen. On fait un travail d'homme, avec des soucis d'hommes. [...] Je suis heureux dans mon métier. Dans un train de banlieue, je sens mon agonie bien autrement qu'ici! J’ai joué, j’ai perdu, c'est dans l'ordre.»

Et ces autres phrases fortes. "Si tu diffères de moi, mon frère, loin de me léser, tu m'enrichis.» Formidable formule à l'heure où le racisme ne montre pas de signe de faiblir. Et, souvent citée: «Nous n'héritons pas de la terre de nos parents, nous l'empruntons à nos enfants.» 